\title{
Investigar la calidad de vida en Argentina
}

\author{
Graciela Tonon*
}

\section{Resumen}

El estudio de la calidad de vida remite al entorno material (bienestar social) y al entorno psicosocial (bienestar psicológico). En 1995 se funda la International Society for Quality of Life Studies (ISQOLS) con el objetivo de incentivar el desarrollo de estudios acerca de la calidad de vida a nivel mundial, a partir de estimular investigaciones interdisciplinarias, convirtiéndose en un forum internacional para los investigadores de diferentes partes del planeta dedicados al tema. En la actualidad se define a la Calidad de vida incluyendo las circunstancias y las percepciones de las personas, sus sentimientos y reacciones a esas circunstancias, existiendo índices que combinan mediciones objetivas y subjetivas. En el caso de Argentina, a partir del año 2004, se organizó en la Facultad de Ciencias Sociales de la Universidad Nacional de Lomas de Zamora el Programa de Investigación en Calidad de vida que forma parte de la red internacional de investigación con el objetivo de desarrollar proyectos en las distintas áreas de estudio de la calidad de vida, tendientes a ser considerados por los organismos estatales de generación de políticas públicas, como una información innovadora a las mediciones de bienestar social de tipo tradicional.

Palabras clave: calidad de vida, bienestar subjetivo, políticas públicas

\section{Abstract}

Researching quality of life in Argentina

The study of quality of life considers at the same time objective and subjective circumstances of people life. In 1995 was created the International Society for Quality of Life Studies (ISQOLS) whose purposes are to promote and encourage research in the field of quality-of-life studies and provide an organization through which all academic and professional researchers interested in QOL studies may coordinate their efforts to advance the field of QOL studies within various disciplines. In the case of Argentina since 2004, the Facultad de Ciencias Sociales, Universidad Nacional de Lomas de Zamora has organized the Research Program in Quality of life to develop different kind of projects about quality of life in different fields, trying to be considered by national institutions that decide public policies.

Key words: Quality of life, life satisfaction, wellbeing, public policies,

\footnotetext{
* Doctora en Ciencias Políticas. Miembro de la International Society for Quality of Life Studies- Programa de Investigación en Calidad de vida. Universidad Nacional de Lomas de Zamora. Argentina. gracielatonon@hotmail.com
} 


\section{Delimitaciones y avances en el estudio de la calidad de vida.}

Los estudios sobre calidad de vida se inician formalmente con los trabajos de Campbell, Converse y Rodgers quienes en los inicios de la década del 70, editaron el texto The quality of american life: percepctions, evaluation and satisfactions (1976). Ya en 1974 Easterlin, había relacionado la economía con el bienestar personal y la felicidad, al expresar que la prosperidad económica de un país no tenía necesariamente consecuencias en la apreciación de la calidad de vida de cada sujeto. En 1979 Morris, a partir del trabajo que desarrolló en el Consejo de Desarrollo de Ultramar, propuso un index de calidad de vida física (ICVF) que examinaba las variaciones a lo largo del tiempo considerando tasas nacionales de mortalidad infantil, expectativa de vida y tasa de alfabetismo en adultos. En el mismo año Estes construyó el denominado "índice de vulnerabilidad social nacional” que luego se convirtió en 1985 en el "índice de progreso social” (IPS), para medir la adecuación en la prestación de servicios sociales, es decir el punto hasta el cual las necesidades sociales básicas eran satisfechas en una nación. De esta manera, se puede decir que, la propuesta teórica de la calidad de vida surgió como respuesta reconceptualizada al tradicional concepto de bienestar.

Pero los orígenes del término se remontan a 1932, cuando el economista inglés Arthur Pigou, pionero de la economía del bienestar, se refirió a cuantificar los servicios o costos sociales de las decisiones de gobierno para poder calcular un producto social neto utilizando por primera vez el concepto calidad de vida. Su obra más conocida fue “The Economics of Welfare” publicada en 1920. Desde ese entonces varias décadas transcurrieron hasta que se iniciara la construcción de los denominados indicadores psicosociales, en forma alternativa al sistema que se venía utilizando de indicadores económicos.

En 1995 se funda la International Society for Quality of Life Studies (ISQOLS) con el objetivo general de incentivar el desarrollo de estudios acerca de la calidad de vida a nivel mundial. Este organismo internacional estimula las investigaciones interdisciplinarias en los campos de la psicología, la política, lo social, la medicina y otras disciplinas dedicadas al desarrollo y el medio ambiente. La ISQOLS se ha convertido en un foro internacional para los investigadores de diferentes partes del planeta dedicados al tema, con el objetivo de coordinar sus esfuerzos de cooperación a fin de desarrollar teoría, métodos de medición y programas de intervención para mejorar la calidad de vida.

En el año 1998 la ISQOLS elabora un documento, resultado de la discusión entre los expertos en el tema, que concluye con el reconocimiento de que la calidad de vida puede ser medida a nivel subjetivo y objetivo. En el citado documento se propone una clasificación de los investigadores en calidad de vida (Sirgy,1998) teniendo en cuenta el nivel de análisis y la unidad de análisis con las cuales trabajan, resultando entonces la identificación de:

a. quienes investigan a nivel macro estudiando la realidad de los países,

b. los dedicados al trabajo en comunidades o regiones geográficas determinadas, 
c. quienes estudian las particularidades en grupos específicos (por ej: niños, mujeres, etc.),

d. los que focalizan su trabajo en la esfera familiar,

e. los dedicados al trabajo en la esfera personal.

Teniendo en cuenta estas clasificaciones se han venido organizando las mesas temáticas que se desarrollan en las Conferencias Internacionales que ha organizado la ISQOLS, en distintos lugares del planeta: Charlotte (USA-1997), Williamsburg (USA1998), Girona (España-2000), Washington (USA-2001), Frankfurt (Alemania-2003), Filadelfia (USA-2004) Grahamstown (Sud Africa-2006), la próxima tendrá lugar en San Diego (USA-2007).

En el escenario de la investigación en habla hispana se destaca en España el trabajo del Dr. Ferrán Casas quien en 1997 organizó el Instituto de Investigaciones sobre Calidad de vida (IRQV) en la Universidad de Girona, con los objetivos de potenciar y desarrollar la investigación en el ámbito del bienestar y de la calidad de vida con un carácter interdisciplinario. Casas (1997) conceptualiza la calidad de vida como función del entorno material y del entorno psicosocial, proponiendo profundizar en el conocimiento tanto de las condiciones materiales de vida como de las percepciones, evaluaciones y aspiraciones de las personas que acompañan su "bien-estar” personal y social; para el conjunto de los ciudadanos de un territorio así como para los miembros de diferentes colectivos específicos. Define calidad de vida como la noción que reconoce entidad a la experiencia que las personas tienen de sus propias formas y condiciones de vida, dando tanto o más valor a esa experiencia que a las condiciones materiales u objetivables definidas como adecuadas por los expertos (Casas,1996).

\section{Acerca de la operacionalización de la teoría}

La operacionalización es un proceso por el cual se traduce el concepto teórico de una variable para hacerla mensurable (Korn,1971). De esta manera y desde la definición que conforma la representación literaria del concepto, se avanza sobre su especificación, dado que todo concepto tiene dimensiones, para finalmente arribar a la construcción de indicadores. (Lazarsfeld,1973). En el caso de la propuesta teórica de la calidad de vida, se revisará, en primer lugar, algunas de las conceptualizaciones teóricas para luego abordar su operacionalización.

Para Casas (1996) la calidad de vida se refiere a las percepciones, aspiraciones, necesidades, satisfacciones y representaciones sociales que los miembros de todo conjunto social experimentan en relación a su entorno y la dinámica social en que se encuentran inmersos, incluyendo los servicios que se les ofrecen y las intervenciones sociales de las que son destinatarios y que emanan de las políticas sociales.

Diener (2005) señala que los dominios de satisfacción son los juicios que las personas hacen para evaluar distintas áreas de su vida, resultando necesario para comprender el nivel de satisfacción que cada persona tiene en cada área bajo estudio, identificar 
previamente el nivel de importancia que reviste para la misma. De esta manera la calidad de vida se refiere generalmente al grado en que la vida de una persona resulta deseable o indeseable, con énfasis en componentes externos tales como los recursos económicos y el medio ambiente.

Ferris (2006) identifica dos fuerzas que producen la calidad de vida: las endógenas y las exógenas. Las endógenas incluyen las respuestas del sujeto ante sus condiciones de vida a nivel mental, emocional y fisiológico. Las exógenas, la estructura social, las influencias sociales, culturales y del medio ambiente, considerando la estructura social en términos de las características demográficas, instituciones, pautas culturales y características psicosociales de la comunidad. En este sentido se ha investigado que el hecho de participar en actividades comunitarias es una fuente de satisfacción; a tal fin Sirgy y Cornwell (2001) exploraron la satisfacción obtenida por los sujetos a partir de la interacción con instituciones de la comunidad, mostrando que la satisfacción con estas instituciones era productora de satisfacción general con la comunidad.

En relación a la operacionalización de la teoría, las propuestas han sido diversas. En esta oportunidad se considera el trabajo de Bob Cummins ${ }^{1}$ quien desde 1994 ha señalado que para estudiar la calidad de vida, resulta necesaria la utilización de indicadores sociales subjetivos, ya que los indicadores denominados objetivos, en general no funcionan como buenos predictores de la calidad de vida subjetiva. En 1994 y junto con McCabe, Romeo and Gullone construyeron la escala de comprensión de calidad de vida (ComQol), operacionalizando el estudio en dominios y considerando el eje objetivo y el subjetivo.

En el caso del bienestar subjetivo, el mismo ha sido medido por preguntas dirigidas hacia los sentimientos de las personas respecto de sí mismos existiendo diferentes formas de hacerlo. Escalas con un solo item que en general se centran en la pregunta acerca de cuan satisfecho se siente la persona con su vida en general. Escalas multi-items, entre las cuales se encuentran las escalas de construcción individual que combinan varios ítems que remiten a la satisfacción global con la vida como el SWLS, (Satisfaction with life scale- Diener et al, 1985) instrumento que contiene 5 dimensiones que miden los juicios cognitivos que una persona tiene sobre su vida en una escala del 1 al 7 en la cual 1 significa "desacuerdo totalmente” y 7 significa "acuerdo totalmente” y las escalas de dominios en relación a la vida que adoptan una representación de la vida en general a partir de dominios (aspectos) de la vida como el PWI (Personal wellbeing indexCummins, 2001) escala del 1 al 10 que mide dominios en relación a la vida.

La medida del bienestar subjetivo es la satisfacción/insatisfacción en tanto producto de todos los factores de la personalidad, variables cognitivas y otra variables en interacción (Cummins, 1998) La pregunta acerca de la satisfacción con la vida en general, hace que la persona se conecte con un sentimiento de lo que le está pasando,

1. El Dr. Cummins es presidente electo de la International Society for Quality of Life Studies (20072008), dirige el Australian Center for Quality of Life en la Universidad de Deakin Australia, donde coordina el International Wellbeing Group. 
más que con la realización de una evaluación cognitiva. Esta pregunta resulta un estimativo que requiere repreguntar por los distintos dominios en forma específica, a fin de conocer como cada uno de ellos contribuye en forma positiva o negativa al estado general. Sucede entonces que cuando se pregunta por un dominio en particular, la respuesta resulta menos abstracta, apareciendo la evaluación cognitiva y decreciendo la expresión afectiva (Cummins,2005).

El wellbeing subjetivo (SWB) estudia la relación entre el "mundo objetivo" de objetos concretos y el "mundo privado", subjetivo. De esta manera y analógicamente como la presión arterial mantiene el equilibrio del cuerpo el SWB es controlado y mantenido por un grupo de dispositivos psicológicos que funcionan bajo el control del núcleo afectivo. Este núcleo (core affect) actúa como la temperatura del cuerpo haciendo conciente cuando una experiencia afecta el nivel "normal". Hasta el momento las investigaciones muestran que para los sujetos, los dos amortiguadores más importantes detectados, son en general el dinero y las relaciones personales

En el año 2001 Cummins construyó el personal wellbeing index (PWI) en tanto grupo de dominios definido como el primer nivel de reconstrucción de la pregunta por la vida en general. La construcción de los dominios quedó de esta manera organizada a partir del principio teórico de la "deconstrucción” aplicado a la satisfacción con la vida en general. En este sentido es importante señalar que los dominios no fueron seleccionados desde una teoría, sino que se utilizaron criterios tales como:

- la necesidad de que cada dominio describa un aspecto que responda tanto desde lo subjetivo como desde lo objetivo, teniendo en cuenta que la calidad de vida remite a ambas dimensiones.

- el hecho de que no se incluya la afectividad y de allí que no se considere el concepto de felicidad.

- la cuestión de que cada dominio resulte un indicador variable inequívoco, es decir que no actúe como mediador.

El PWI utiliza una escala del 0 (cero) al 10 (diez) vida en la cual el 0 corresponde a no estar satisfecho y el 10 corresponde al más alto nivel de satisfacción. Los resultados obtenidos se ajustan posteriormente a un orden del 0 al 100, habiéndose establecido que la "medida normal" se encuentra comprendida entre 70 y 80 , si bien las mediciones en algunos países muestran valores por debajo. (Cummins et al, 2003). El instrumento a nivel internacional es conocido como International Wellbeing Index e incluye dos sub-escalas: la personal y la nacional.

Los dominios sirven como dispositivos psicométricos y permiten una medición más refinada de la calidad de vida, de la que puede ser alcanzada con una simple pregunta. Además si la suma de los dominios es equivalente a la satisfacción general con la vida, entonces se puede considerar que ese grupo de dominios es válido en tanto medida de calidad de vida. La discusión se centra en conocer si los dominios son tan centrales para la experiencia de la calidad de vida que pueden ser considerados transnacionales. Si bien algunas investigaciones así lo muestran, Cummins y Lau, a 
partir del año 2002, convocaron a trabajar a investigadores de diferentes lugares del planeta, con el objetivo de lograr la validación trasnacional del index, organizando el International Wellbeing Group (IWG).

EL IWG se encuentra conformado por investigadores de 45 países de distintos continentes: Alemania, Algeria, Argentina, Australia, Bélgica, Canadá, China, Colombia, Croacia, Eslovenia, España, Estados Unidos, Filipinas, Finlandia, Gran Bretaña, Groenlandia, Holanda, Hungría, India, Irán, Irlanda, Israel, Italia, Japón, Laos, Líbano, Malasia, Mauritania, México, Nueva Zelanda, Noruega, Paquistán, Polonia, Portugal, Rumania, Rusia, Ruanda, Singapur, Sudáfrica, Suiza, Taiwán, Tailandia, Turquía, Vietnam del Norte y West Indies. Asimismo el WBI se encuentra traducido hasta el momento a 13 idiomas: alemán, árabe, chino, croata, eslovaco, español, inglés, italiano, noruego, persa, portugués, ruso, y tibetano.

\section{El desarrollo de estudios sobre calidad de vida en Argentina}

En el caso de Argentina, en el año 2004 se organiza en la Facultad de Ciencias Sociales de la Universidad Nacional de Lomas de Zamora el Programa de Investigación en Calidad de vida que forma parte de la red internacional de investigación.

El objetivo general del Programa es el desarrollo de proyectos de investigación en las distintas áreas de estudio de la calidad de vida, desde la mirada de los protagonistas, tendientes a ser considerados por los organismos estatales de generación de políticas públicas, como una información innovadora a las mediciones de bienestar social de tipo tradicional. Son sus objetivos específicos:

a. Desarrollar proyectos de investigación en las distintas áreas de estudio de la calidad de vida, desde la mirada de los protagonistas, tendientes a ser considerados por los organismos estatales de generación de políticas públicas, como una información innovadora a las mediciones de bienestar social de tipo tradicional.

b. Lograr interactuar y colaborar con otros centros de investigación internacional referidos a estudios sobre calidad de vida.

c. Generar un espacio de integración docencia-investigación para alumnos/as de la Facultad de Ciencias Sociales de la Universidad Nacional de Lomas de Zamora.

d. Brindar un espacio de participación e intercambio a investigadores dedicados al tema de otras instituciones del país.

e. Producir publicaciones científicas referidas al tema.

f. Organizar reuniones científicas nacionales e internacionales que convoquen a investigadores en el tema.

Hasta el momento las líneas de investigación que se vienen desarrollando tienen como tema: juventud y calidad de vida y calidad de vida laboral. El Programa organiza Jornadas de investigación en Calidad de vida a la cual concurren investigadores de diferentes países de Latinoamérica: Chile, Venezuela, Colombia, Brasil, Uruguay y equipos de investigación nacionales de la Facultad de Humanidades de la Universidad 
Nacional de Mar del Plata y la Facultad de Ciencias de la Salud de la Universidad Nacional de Salta. Recibe doctorandos-pasantes del Doctorado en Psicología de la Universidad de Palermo y del Doctorado en Ciencias Sociales, Niñez y Juventud de la Universidad de Manizales, Colombia. Dos veces al año se publica el Volumen Calidad de Vida de la Revista Hologramatica (www.hologramatica.com.ar) publicación virtual de la Facultad de Ciencias Sociales de la Universidad Nacional de Lomas de Zamora que se encuentra incluida en el Latindex, en Directory of Open Access Journals (doaj.org) y en Caicyt-Conicet. Como resultado escrito de los trabajos de investigación que se desarrollan en el marco del Programa, se ha publicado en el año 2006 el primer libro titulado Juventud y protagonismo ciudadano.

\section{Conclusiones}

El estudio de la calidad de vida remite al entorno material (bienestar social) y al entorno psicosocial (bienestar psicológico), basado en la experiencia y en la evaluación que cada persona tiene de su situación, incluyendo una visión global de la vida de la persona que se denomina satisfacción vital. Vista como el "buen vivir", la calidad de vida es considerada una vivencia interna no comunicada; y de allí que la idea de estar bien es una referencia a estar por encima de un determinado punto evaluable por debajo del cual se está “mal” (Casas, 1996).

El efecto de las relaciones sociales y de las relaciones con el medio, se encuentra mediatizado por la particular mirada de cada sujeto, es decir la manera en que cada sujeto interpreta su contexto y situación, es por eso que esta mirada es la llave que determina su calidad de vida.(Patrick, Edwards, Topolski y Walwick, 2002)

Actualmente el término es utilizado en forma cotidiana e implicando la participación de los ciudadanos en la evaluación de lo que les afecta, convirtiéndose en un concepto con significación política, que contribuye al estudio del bienestar de las personas, desde la perspectiva del bienestar físico y psicológico, relacionando las necesidades materiales con las socio-afectivas e integrando mediciones psicológicas y sociales de percepción y evaluación de las propias experiencias de los sujetos (Casas,1998).

Los estudios sobre calidad de vida nos presentan la posibilidad de una nueva mirada teórica, tendiente a un trabajo desde las potencialidades más que desde las carencias y con un anclaje comunitario de tipo psicosocial que incluye el análisis del contexto socio político; partiendo de la consideración del entorno material en conjunción con el social, considerando a la persona tradicionalmente llamada “objeto" como "sujeto” y protagonista del accionar. De esta manera la propuesta teórica de la calidad de vida plantea una realidad social y política basada en el respeto de los derechos humanos, generando la necesidad de trabajar en forma integrada (Tonon, 2003). De esta manera, la propuesta teórica de la calidad de vida se constituye como mirada privilegiada que integra las dimensiones macro y micro, intentando que la visión de los protagonistas sea tenida en cuenta a la hora de la decisión de las políticas públicas, al conformarse como una información innovadora respecto de las tradicionales mediciones de bienestar social. 


\section{Bibliografía}

1. Campbell, A., Converse, P., Rodgers, E. (1976) The Quality Of American Life: Perceptions, Evaluations and Satisfactions. New York: Russel Sage Foundation

2. Casas, F. (1996) Bienestar social. Una introducción psicosociológica. Barcelona: PPU.

3. Casas, F. (1999) Calidad de vida y calidad humana. Revista Papeles del Psicólogo $N^{\circ}$ 74. Madrid, Colegio de Psicólogos de Madrid. 46-54.

4. Cummins, R et al. (2003) The australian unity wellbeing index: and overview. En Land, K (editor). SINET $N^{\circ}$ 76. USA, Duke University, 1-4.

5. Cummins, R. (ed) (1998) Quality of life definition and terminology. A discussion document. ISQOLS.

6. Cummins, R., Gullone, E. y Lau, A. (2002).The universality of subjective wellbeing indicators. En Social Indicators Research Vol 16. The Netherlands. Kluwer Academic Publishers, 5-6.

7. Diener, E. (2005) Guidelines for national indicators of subjective well-being and ill-being. Documento de discusión de la ISQOLS.

8. Diener, E, Emmons, R, Larsen, R y Griffin, S (1985). The satisfaction with life scale. Journal of Personality Assesment 49. 71-75.

9. Easterlin, R (2004) A brief history of QOL Studies in Economics. Documento de la Universidad de Southern California.

10. Estes, R (1999) Hacia un índice de calidad de vida: enfoques empíricos para la evaluación del bienestar humano a nivel internacional. En Klisberg, B (comp) Pobreza: un tema impostergable. México: CLAD-Fondo de Cultura Económica, 131-148

11. Ferris, A. (2006) A theory of the social structure and the quality of life. En Applied Research in Quality of Life. Vol 1. Number 1. The Netherlands. Springer. 117-123

12. Korn, F. (1971) El significado del término variable. En Korn, F y otros Conceptos y variables en la investigación social. Buenos Aires: Ediciones Nueva Visión. 9-19

13. Lazarsfeld, P. (1973). De los conceptos a los índices empíricos. En Boudon, R. y Paul Lazarsfeld, P. Metodología de las ciencias sociales, Vol. I. Barcelona: Ed. Laia.

14. Patrick, D, Edwards, T, Topolski, T y Walwick, J. (2002). Youth quality of life: a new measure incorporating the voices of adolescents. En QOL Newsletter $N^{\circ} 28$. Lyon, Mapi Research Institute, 7-8

15. Sirgy, J. y Cornwell, T. (2001) Further validation of the Sirgy et al'measure of community quality of life. Social Indicators Research 56, 125-143 
16. Tonon, G (2005) Apreciaciones teóricas del estudio de la calidad de vida en Argentina. HOLOGRAMÁTICA Año II, Número 2 V 1 (2005).27-49 www.hologramatica.com.ar

17. Tonon, G (2003) Calidad de vida y desgaste profesional. Buenos Aires: Espacio Editorial.

18. Tonon, G. (2006) (comp) Juventud y protagonismo ciudadano. Buenos Aires: Espacio Editorial. 
Psicodebate 8. Psicología, Cultura y Sociedad - Noviembre 2007 